Sarvenaz Kashefi ${ }^{1}$, Ramesh Omranipour ${ }^{2}{ }^{3}$, Habibollah Mahmoodzadeh ${ }^{3}$, Hamid Ahmadi ${ }^{2}$, Abbas Mirshafiey ${ }^{1}$

${ }^{1}$ Department of Immunology, School of Public Health, Tehran University of Medical Science, Tehran, Iran

${ }^{2}$ Breast Cancer Research Center (BDRC), Tehran University of Medical Science, Tehran, Iran

${ }^{3}$ Department of Surgical Oncology, Tehran University of Medical Science, Tehran, Iran

\title{
Clinical improvement of diabetes mellitus type 1 by $\beta$-D-mannuronic acid (M2000) in a breast cancer patient - as a case report
}

\section{ABSTRACT}

A 56 years old female with breast cancer (BC) and poor controlled diabetes mellitus type 1 (DM1) which has registered in a clinical trial for assessment of therapeutic efficacy of $\beta$-D-mannuronic acid (M2000) on pre-surgical $\mathrm{BC}$ patients is described in this case report. After receiving M2000, the patient was followed for 9 weeks. During this period, cancer mass details, fasting blood glucose (FBG) levels, 2-hour post-prandial blood glucose (2HPP), blood uric acid (BUA) level and urine analysis (UA) were continuously controlled. After 9 weeks of treatment with M2000, her FBG, BUA and UA decreased significantly. This finding was exactly in accordance with our published experimental data about the anti-diabetic effect of M2000 in an animal model. Therefore, it might be concluded that M2000 is probably able to improve DM1 by reducing FBG level, BUA level, glycosuria, ketonuria and proteinuria. (Clin Diabetol 2019; 8, 4: 227-229)

Key words: $\beta$-D-mannuronic acid, M2000, breast cancer, diabetes mellitus, NSAID

Address for correspondence:

Dr. Habibollah Mahmoodzadeh,

Prof. Abbas Mirshafiey

Tehran University of Medical Science, Tehran, Iran

Phone: +982161192541, fax: +982188485429

Phone, fax: +982188954913, e-mail:

e-mail: hmahmoodzadeh@tums.ac.ir;

mirshafiey@tums.ac.ir

Clinical Diabetology 2019, 8, 4, 227-229

DOI: $10.5603 /$ DK.2019.0018

Received: 08.02.2019

Accepted: 08.04.2019

\section{Introduction}

BC patients with DM1 have poor prognosis with unknown mechanism [1]. Non-steroidal anti-inflammatory drugs (NSAIDs) are among the drugs prescribed for $B C$ and DM1 [1]. However, their excessive use can be associated with adverse effects in the gastrointestinal tract, which limits their use in certain cases [2].

Alginates are linear copolymers found in brown seaweed and consist of $\beta$-D-mannuronic acid (M-block) and $\alpha$-L-guluronic acid (G-block) which are bound together by 1,4 glycoside bonds. M2000 (Patent PCT/ /EP2017/067919) is a low molecular weight small molecule $\left(\mathrm{C}_{6} \mathrm{H}_{10} \mathrm{O}_{7}\right)$ which is produced from sodium alginate, based on the protocol of Mirshafiey, et al. The purity of the M2000 has been confirmed using Carbon-13 Nuclear Magnetic Resonance ( $\left.{ }^{13} \mathrm{C}-\mathrm{NMR}\right)$ and Fourier Transform Infrared Spectroscopy (FT-IR) [3]. Some data showed M2000 is a NSAID with a prominent immunosuppressive feature which can be used safely [4]. The molecular mechanism of therapeutic efficacy of this novel drug is based on its inhibitory effects on matrix metalloproteinase 2, 9 (MMP2, 9) activity, decrease in immune cells infiltration in inflammatory foci, blocking the toll-like receptor 2, $4($ TLR2, 4) downstream signaling transduction pathway, reduction of the level of inflammatory cytokine such as interleukin (IL)- 6 and tumor necrosis factor (TNF)- $\alpha$, a decrease in antibody production and induction of apoptosis using fibrosarcoma cell line $[5,6]$. In this study, another effect of M2000 in the treatment of DM1 was presented for the first time as a case report in an ongoing phase I and II clinical trials on Iranian BC patients. In this report, a 56-year-old patient diagnosed with $B C$ and poor controlled DM1 is introduced. 
Table 1. Laboratory data changes during treatment with M2000

\begin{tabular}{|c|c|c|c|c|}
\hline Parameter & $\begin{array}{c}\text { A day before } \\
\text { treatment }\end{array}$ & $\begin{array}{c}3^{\text {rd }} \text { week after } \\
\text { treatment }\end{array}$ & $\begin{array}{c}6^{\text {th }} \text { week after } \\
\text { treatment }\end{array}$ & $\begin{array}{c}9^{\text {th }} \text { week after } \\
\text { treatment }\end{array}$ \\
\hline $\mathrm{FBG}[\mathrm{mg} / \mathrm{dL}]$ & 189 & 122 & 101 & 91 \\
\hline $2 \mathrm{HPP}[\mathrm{mg} / \mathrm{dL}]$ & 260 & 246 & 223 & 196 \\
\hline Uric acid [mg/dL] & 8.2 & 7.6 & 6.3 & 5.8 \\
\hline Proteinuria [mg/dl] & 321 & 255 & 208 & 117 \\
\hline Glycosuria [mmol/l] & 1.9 & 1.5 & 1.2 & 1 \\
\hline Ketonuria $[\mathrm{mmol} / \mathrm{l}]$ & 2.3 & 1.4 & 1 & 0.7 \\
\hline
\end{tabular}

FBG - fasting blood glucose; 2 HPP -2 hour post-prandial

\section{Case report}

A 56 years old female with the body mass index of $30.1 \mathrm{~kg} / \mathrm{m}^{2}$ (height: $164 \mathrm{~cm}$, weight: $81 \mathrm{~kg}$ ) patient with $B C$ that refused surgery and chemotherapy selected. She accepted treatment with M2000 for BC. She also had uncontrolled DM1 diagnosed 8 years ago, receiving 68 units of Lantus and Novorapid insulin daily (24 units in the morning, 22 units in the afternoon and 22 units at night). Mean daily glycaemia was $206 \pm 15$ $\mathrm{mg} / \mathrm{dL}$. Patient had no comorbid diseases nor diabetic complications. Treatment with M2000 was started at a dose of $1000 \mathrm{mg}$ per day for 9 weeks to evaluate its effect on breast tumor growth. Laboratory data changes are shown in Table 1. The patient did not report side effects during the treatment.

\section{Discussion}

The dysregulated metabolism in poorly controlled diabetes causes a long-term pro-inflammatory condition characterized by increased levels of IL-6, TNF- $\alpha$, $\mathrm{C}$-reactive protein (CRP) and other markers of chronic inflammation [2]. Recent evidence suggested that, persistent inflammation may contribute to genetic instability and chronic forms of inflammation can increase the risk of cancer [1]. This finding is also confirmed by credible evidence regarding the reducing effect of NSAIDs on the risk of certain types of cancer $[1,6]$.

The mechanism of inflammation in diabetic patients is unclear. Chronic oxidative stress is associated with chronic inflammation. Oxidants affect almost all stages of the inflammatory response process, including the release of inflammatory cytokines, sensing by innate immune receptors of TLRs, nucleotide-binding oligomerization domain-like receptors and activating the triggering signaling of the adaptive cellular response to such signals. Reactive oxygen species may damage lipids, proteins and DNA and then begin carcinogenesis [2]. In addition, chronic inflammation is associated with the increased levels of TNF- $\alpha$, which strongly activates nuclear factor kappa B (NF- $\kappa$ B) and enhances the downstream signaling transduction, ultimately leading to progression

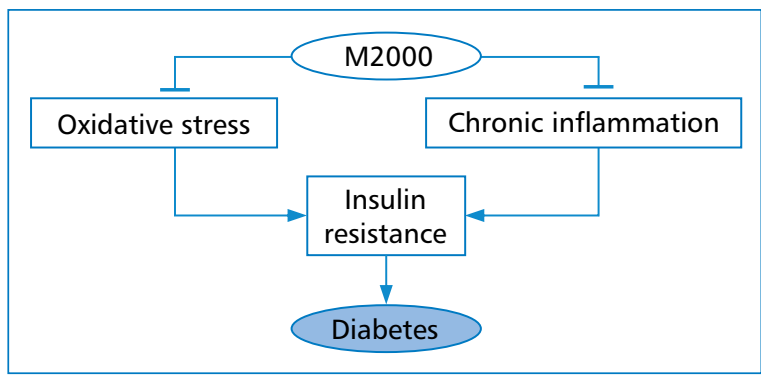

Figure 1. Schematic hypothesis: the anti-diabetic effect of M2000

of many tumors [7]. NF- $\kappa$ B protein complex contributes to the proliferation and survival of malignant cells, increases angiogenesis, metastasis, damages adaptive immunity and mediates the response to hormones and chemotherapy drugs. Therefore, long-term exposure to chronic inflammation and oxidative stress results in conversion of susceptible cells to malignant cells [8].

The main objective of this study was to improve BC by reducing tumor growth using M2000. M2000 was introduced as a novel NSAID with immunosuppressive effects due to its impact on the expression and activity of cyclooxygenase 1, 2 (COX1, 2) genes. In terms of the molecular mechanism, this new drug exerts its therapeutic effect through inhibiting the activity of MMP2 and MMP9, reducing the infiltration of immune cells in inflammatory foci, decreasing the level of inflammatory cytokines IL6 and IL17, reducing antibody production and inducing apoptosis using fibro sarcoma cell line. The results of a study showed that, this new NSAID can affect the M2000-treated mice through decreasing the expression levels of blood glucose, Scavenger receptorA (SR-A), Lipoxygenase-1 (LOX-1), CD36 and CD68 compared to untreated diabetic rats (Figure 1) $[9,10]$.

Collectively, after 9 weeks of follow-up, there was a significant improvement in the level of FBG, 2HPP and UA in the patient with DM1. Therefore, it might be concluded that M2000 is a new NSAID that can improve DM1 in patients with BC. 


\section{Conflicts of interest}

The authors report that they have no conflicts of interest.

\section{Acknowledgments}

The authors would like to thank Tehran University of Medical Science.

\section{REFERENCES}

1. Zhao XB, Ren GS. Diabetes mellitus and prognosis in women with breast cancer: A systematic review and meta-analysis. Medicine (Baltimore). 2016; 95(49): e5602, doi: 10.1097/ MD.0000000000005602, indexed in Pubmed: 27930583.

2. Xu CX, Zhu HH, Zhu YM. Diabetes and cancer: Associations, mechanisms, and implications for medical practice. World J Diabetes. 2014; 5(3): 372-380, doi: 10.4239/wjd.v5.i3.372, indexed in Pubmed: 24936258.

3. Fattahi MJ, Abdollahi M, Agha Mohammadi A, et al. Preclinical assessment of $\beta$-d-mannuronic acid (M2000) as a non-steroidal anti-inflammatory drug. Immunopharmacol Immunotoxicol. 2015; 37(6): 535-540, doi: 10.3109/08923973.2015.1113296, indexed in Pubmed: 26584020.

4. Mirshafiey A, Taeb M, Mortazavi-Jahromi SS, et al. Introduction of $\beta$-d-mannuronic acid (M2000) as a novel NSAID with immunosuppressive property based on COX-1/COX-2 activity and gene expression. Pharmacol Rep. 2017; 69(5): 1067-1072, doi: 10.1016/j.pharep.2017.04.015, indexed in Pubmed: 28951072.
5. Mirshafiey A, Cuzzocrea S, Rehm BHA, et al. M2000: a revolution in pharmacology. Med Sci Monit. 2005; 11(8): PI53-PI63, indexed in Pubmed: 16049391.

6. Hosseini F, Hassannia H, Mahdian-Shakib A, et al. Targeting of crosstalk between tumor and tumor microenvironment by $\beta$-D mannuronic acid (M2000) in murine breast cancer model. Cancer Med. 2017; 6(3): 640-650, doi: 10.1002/cam4.1013, indexed in Pubmed: 28211615.

7. Onitilo AA, Engel JM, Glurich I, et al. Diabetes and cancer I: risk, survival, and implications for screening. Cancer Causes Control. 2012; 23(6): 967-981, doi: 10.1007/s10552-012-9972-3, indexed in Pubmed: 22552844.

8. M Farahani M, Motevaseli E, Maghsood F, et al. Anti-inflammatory property of $\beta$-D-Mannuronic acid (M2000) on expression and activity of matrix metalloproteinase-2 and -9 through CD147 molecule in phorbol myristate acetate-differentiated THP-1 cells. Iran J Allergy Asthma Immunol. 2017; 16(5): 443-451, indexed in Pubmed: 29149784.

9. Mortazavi-Jahromi SS, Alizadeh S, Javanbakht $\mathrm{MH}$, et al. Antidiabetic effect of $\beta$-D-mannuronic acid (M2000) as a novel NSAID with immunosuppressive property on insulin production, blood glucose, and inflammatory markers in the experimental diabetes model. Arch Physiol Biochem. 2018 [Epub ahead of print]: 1-6, doi: 10.1080/13813455.2018.1481094, indexed in Pubmed: 29882437.

10. Mortazavi-Jahromi SS, Alizadeh S, Javanbakht $\mathrm{MH}$, et al. Cardioprotective effect of $\beta$-d-mannuronic acid (M2000) as a novel NSAID on gene expression of oxLDL scavenger receptors in the experimental diabetic model. Immunopharmacol Immunotoxicol. 2018; 40(4): 284-289, doi: 10.1080/08923973.2018.1455209, indexed in Pubmed: 29619884. 
